\title{
A Review of Image Processing Software Techniques for Early Detection of Plant Drought Stress
}

\author{
Chege A. Kirongo \\ Computer Science and IT Department \\ Meru University of Science and Technology \\ Meru, Kenya
}

\begin{abstract}
Water stress is one of the most important growth-limiting factors in crop production around the world, water in plants is required to permit vital processes such as nutrient uptake, photosynthesis, and respiration. Drought stress in plants causes major production losses in the agricultural industry worldwide. There is no sensor commercially available for real-time assessment of health conditions in beans. Currently, there are several methods to evaluate the effect of water stress on plants and commonly practiced method over the years for stress detection is to use information provided by remote sensing. Studies exist which determined the effect of water stress in plants grown under the different watering regime, while other studies explore the performance of the artificial neural network techniques to estimate plant yield using spectral vegetation indices. This review recognizes the need for developing a rapid cost-effective, and reliable health monitoring sensor that would facilitate advancements in agriculture
\end{abstract}

Keywords: Image Processing, Artificial Neural Networks, Drought stress, Algorithm, Technique

\section{INTRODUCTION}

Water stress is one of the most important growth-limiting factors in crop production around the world. Water in plants is required to permit vital processes such as nutrient uptake, photosynthesis, and respiration. Drought stress in plants causes major production losses in agriculture industry worldwide. Monitoring of health and detection of the draught in plants and trees is critical for sustainable agriculture.

To the best of my knowledge, there is no sensor commercially available for real-time assessment of health conditions in beans. Currently, there are several methods to evaluate the effect of water stress on plants. A promising and commonly practiced method over the years for stress detection is to use information provided by remote sensing. The adaptation of remote sensing and other non-destructive techniques could allow for early and spatial stress detection in vegetables.

Studies exist which determined the effect of water stress in plants grown under the different watering regime. Other studies explore the performance of the artificial neural network techniques to estimate plant yield using spectral vegetation indices. It describes the currently used technologies that can be used for developing a ground - based sensor system to assist in monitoring health and draught in plants under field conditions.

These technologies include normalization difference vegetation index, leaf temperature and microclimatic information, automatic segmentation for scanned images to visualize features by specific color hues and intensities. The method involves classification of pixels, determined by leaf regions with or without color. Artificial Neural Network is applied in a self - organizing Kohonen network and a linear perceptron output layer. Other researchers have used ANOVA analysis of the timing of draught stress detection in plants.

\section{BACKGROUND}

In order to assess the vitality of a plant, it is necessary to capture the required features which describe the plant's state. For instance, the bending angle of the leaves towards the ground might be a measure of the drought stress level of the plant. The reason is the loss of turgor pressure caused by the decline of water in the leaves. This effect forces the leaves to bend down, due to gravity. Features like this can be used in biological models in order to describe how certain feature values correspond to the health state of a plant. Unfortunately, relevant features are often difficult to measure using remote systems. There are two main difficulties:

There are two main difficulties:

Firstly, the measurement system must provide sufficient data for the extraction of the features. For example, a single 2D image of a bean plant taken by a simple digital camera would not be able to obtain information about the bending angles since most leaves would be in a position where it is impossible to capture the required information. Important key points like the tip or the stem of a leaf might not be visible in all cases due to an unfavorable point of view or overlapping leaves. Taking several pictures from different points of view might solve this problem but raises others, e.g. how to identify same leaves in different images.

Secondly, the features must be extracted from the measurement data. Thus, potent imaging algorithms are required which are able to detect relevant parts of the plant and infer complex features from the measurement data. However, this is not a trivial task since the data is often very complex. In the case of laser scanners, the measurement data might consist of point clouds with thousands of 3D points. Thus, the measurement data can be very large and full of redundancies.

Nevertheless, some relevant information might be missing, e.g. because of occlusions or other negative effects.

\section{IMAGE PROCESSING TECHNIQUES}

\subsection{Existing Techniques}

There exist several methods for plant measurement. In [1], a short overview of several methods to acquire structural data is given: There are contact measurement systems, for instance, magnetic digitizers, which consist of a signal receiver and pointer, allowing the user to record the $3 \mathrm{D}$ spatial coordinates of the pointer within a [certain] hemisphere [...] from the receiver" [1, p. 17]. Nevertheless, magnetic digitizers are 
prone to disturbances caused by iron, which might be a problem in greenhouse environments.

To overcome this problem, sonic digitizers can be used. However, those are sensitive to wind fluctuations and are therefore not suited for applications in the field [1, p. 17].

Besides this contact measurement systems, there is also a big range of non-contact measurement systems. Apart from a sheet of light laser scanning, which was used for this thesis and is therefore explained further in section 3.1, there exist systems based on time of light, volumetric intersection, stereo vision and other methods [1, p. 17_]

Time of light systems works by emitting a laser beam which is backscattered by the plant. The returning beams are then captured via a photodiode in the receiver optics. The distance between the plant and the optics can then be estimated by measuring the time interval between emitting and receiving the laser beam. In volumetric intersection systems, the surface of a plant is captured by recording the silhouette of the plant against a monochromatic background, which is then discarded. By turning the plant and taking silhouettes from different angles, the 3D surface of the plant's outline can be reconstructed. Obviously, this method has problems with occlusions or overlapping plant parts.

Stereo vision systems use two cameras, positioned at a distance, which record the same scene. From the shift of corresponding points in both views, the $3 \mathrm{D}$ position of these points in real-world coordinates can be computed.

All of the mentioned systems are used to capture the surface and therefore the architecture of a plant. To acquire data about functional aspects of plant growth, for instance, water transport or pigment content, other imaging techniques are used.

Chaerle [2] gives an overview of such imaging techniques which are used to monitor plant health. Those include fluorescence, luminescence, thermal imaging, magnetic resonance and reactance measuring. These imaging techniques are already proven to be useful for vitality assessment.

Please use a 9-point Times Roman font, or other Roman font with serifs, as close as possible in appearance to Times Roman in which these guidelines have been set. The goal is to have a 9-point text, as you see here. Please use sans-serif or non-proportional fonts only for special purposes, such as distinguishing source code text. If Times Roman is not available, try the font named Computer Modern Roman. On a Macintosh, use the font named Times. Right margins should be justified, not ragged.

\subsection{Plant Stress Detection Techniques}

As it was shown in the previous section, there exists a wide range of different imaging systems, each being useful for different data acquisition tasks. However, further techniques are necessary to detect valuable information in the data and extract it reliably. Depending on the task, each has a varying degree of difficulty, which is illustrated in the following examples.

In the experiments described by Romer et al. [3], hyperspectral images of barley and corn plants have been analyzed in order to detect drought stress in a very early stage, i.e. before the plant is damaged. The experiments have been performed on the level of single plants in the case of barley, whereas corn plants were measured directly in the field.
The data consisted basically of histograms of the hyperspectral plant images. To assess the vitality state of the plants, Romer et al. used an unsupervised classification method called simplex volume maximization. The key idea of this method is to find archetypal samples in the data which represent the most extreme cases of plant growth, e.g. very healthy or very stressed plant samples.

This is achieved by comparing the distances between all data points and declaring the samples as archetypes which span the greatest volume of the distance around all other samples. Afterwards, all non-archetypal samples can be expressed as a convex combination of the archetypes, where the coefficients relate to the similarity of a data sample to a respective archetype. The benefit of this is that the resulting classes can be interpreted by humans although they were learned using an unsupervised learning technique. Nevertheless, at least with respect to the barley dataset, a human expert was necessary in order to assert the correctness of the archetypes.

The method was compared to visual classification and to the usage of vegetation indices, e.g. calculating the ratio of ground which is covered by biomass. Romer et al. achieved good results. In the barley experiment, stress was detected 5 days earlier than with visual classification and 4 days faster than with the tested vegetation indices.

As Romer et al. [3, p. 879] mention, despite several laboratory studies that have shown a relationship between the amount of water in the leaf and the spectral reactance in the optical region [4], at canopy level the determination of water content presents some difficulties, mainly due to the large reactance variation among leaves with the same water status [5], structural changes associated with loss of turgor [6] or small reactance differences at different levels of water stress".

Therefore, it is difficult to detect drought stress in single plants using reactance imaging.

Moreover, since the category of plants is grown to be used for medical products, it is necessary to control the state of every single plant regularly and very often, e.g. every day. In the corn experiment of Romer et al., however, the measurement system took one minute to acquire an image of the size _ $2 \times 1.5 \mathrm{~m}$, which is quite slow if a large field with hundreds of plants has to be processed.

\section{DISCUSSION}

\subsection{State of the Art in Plant Drought Stress Algorithms}

A different kind of approach is taken by Seatovic [10], who presents a system for the recognition and treatment of broadleaved dock, which is a highly competitive and persistent sort of weed. This system uses an infrared laser scanner and a high-resolution smart camera which are mounted on a carrier vehicle. Besides the sensor system the vehicle carries an herbicide spraying component which is able to treat certain parts of the captured area, if a weed plant is detected. The vehicle can be moved over a field with a speed of $1 \mathrm{~ms} 1$ while weed plants are detected and treated in real-time. In the image data, weed plants are identified by finding contiguous surface patches. This is done with a simple edge detection algorithm $[10$, p. 174f]. The surface patches are then compared to objects of a plant database with respect to simple features like shape and texture. The main benefit of Seatovic's approach is that features like shape can be derived from the raw measurement data. Therefore, they can be computed fast 
enough to be used in a real-time system. Nevertheless, he reports that only $62-91 \%$ of the weed plant surface have been detected in field tests. This detection rate was sufficient in his project since the radius of the herbicide spraying nozzles was big enough to treat all weed plants even if not all leaves have been detected.

However, other application contexts might require a higher accuracy. For example, a system for treatment of vermin might need higher accuracy rates since any missed bugs could spread across already treated plants.

Both approaches report good results on the level of whole plant stands, whereas a reliable detection of single leaves is difficult. A reason might be that both approaches use quite simple features. Although they differ in terms of data acquisition and feature extraction methods, both approaches use simple features which rather describe the images instead of the plants which are depicted.

Consequently, it is difficult to derive information about certain plant parts like leaves since they are on a higher level of detail. If that information is required, further advanced feature extraction techniques are necessary. Unfortunately, the segmentation of image data to corresponding plant parts and the automatic calculation of certain features is a challenging task due to its complexity. As van der Heijden [11, p. 19, p. 22] stated, "automatic feature extraction is still in its infancy" and consequently "plant features still have to be extracted interactively since current software is not yet capable of fully automatic extraction of plant features in complex images".

Since an advanced feature extraction is the bottleneck of many applications, there are some recent approaches to a solution. Paulus et al. [12] provide an algorithm to classify parts of point cloud data, e.g. to distinguish leaves from stems. Their approach is based on surface feature histograms, which consist of local geometric point features, e.g. neighborhood characteristics. By differentiating among different classes of histograms, different plant parts can be detected in the point cloud. They confirmed their methods by successfully applying them on grapevine and wheat plant organs, where classification accuracy rates up to $98 \%$ could be reached.

In the work of Balfer [13], a skeletonization algorithm is explained, which uses semantic annotations to extract a precise 3D model of complex grapevine stem systems. From this model, structural features can be extracted, e.g. the lengths of the peduncles. However, the approach requires expert knowledge in order to find and model suitable semantic annotations

\subsection{Existing Methodological Approaches}

Dornbusch et al. [14] propose a method to extract morphological traits of a plant, e.g. width and length of stem segments and leaves by fitting an architectural model to point cloud data. This method is quite promising since it provides meaningful features which can be interpreted by human experts. However, a crucial step, namely segmenting the point cloud of a whole plant into smaller segments (i.e. the actual detection), is still performed manually in Dornbusch's approach.

Camargo [15] uses histogram-based approaches to segmenting 2D images of cotton plants, e.g. to find spots which were damaged by bugs or infected with diseases. The approach works well and is feasible, nevertheless, it is only applicable to $2 \mathrm{D}$ image data and is therefore not suitable to extract 3D features like bending angles of leaves.
Given that meaningful features of plant growth can be extracted from sensor data, the actual assessment of plant vitality can be performed by assigning certain classes or measures of plant health to patterns or constellations of attribute values. In many agricultural applications, standard machine learning techniques are used to deal with this kind of task.

Mucherino [16] provides a survey of how techniques such as k-nearest neighbor, artificial neural networks or support vector machines have been successfully applied to solve agricultural problems. The fields of interest include prediction of wine quality, soil quality analysis, recognition of pig sounds and the detection of meat and bone meal in feedstuffs for animals. Unfortunately, stress classification of plants is none of the discussed problems.

In the field of plant classification, support vector machines are widely used. Rumpf et al. [18] use support vector machines to distinguish diseased from non-diseased hyperspectral sensor data of sugar beet leaves. They mainly use simple features like vegetation indices, i.e. the ratio of ground which is covered by biomass. However, since it is based on hyperspectral data, their approach allows for early disease detection.

Camargo [9] uses support vector machines to classify visual symptoms of cotton plant diseases, i.e. visual damages of the leaves. As it was already mentioned, his approach relies on 2D images which are searched for relevant segments using color intensity histograms. Therefore, the approach is only feasible if no other information, e.g. 3D geometry, is required.

\subsection{Approaches for Morphological Design of Leaf Model}

Most of the mentioned approaches tend to avoid the bottleneck of feature extraction by reducing it to a minimum, either by using global image data like spectral histograms or by only extracting simple features like texture, color or width and length values of simple shapes. However, those simple features only describe visual properties of the images but lack a semantic relation to the growth behavior of the plants which are depicted.

More desirably, the features should correspond directly to information which is interesting for a human expert. Therefore approaches that links to the work of Uhrmann et al. [5], in which a morphological leaf model should be studied to extract feasible growth parameters. The difference to other approaches is that the model should consider complex features, e.g. attributes describing the bending behavior of the leaf along certain axes. In contrast to simple attributes like length or width of the leaf, this complex attributes should be directly correlated with the vitality state of the plant.

\section{CONCLUSION}

Since using a leaf model is a very new technique, a model should be validated so as to analyse the leaf model features and to show which of them are relevant for the description of drought stress with respect to plants.

Furthermore, classification techniques should be examined for the evaluation of the model leaf features. Many of the mentioned approaches use support vector machines for the classification process since they provide excellent results in most cases. However, there are different classification techniques which are easier to interpret by human experts. For example, decision trees or linear regression provide an easier model in terms of comprehensiveness. 
Therefore, research to evaluate several classifiers with respect to the task of learning the features provided by the leaf model can be considered. For instance, if decision trees turn out to provide classification results comparable to support vector machines, they should be preferred in a practical application, since they are easier to evaluate by non-technical experts, e.g. biologists.

Therefore, a model for the impact of drought stress on plant growth can be inferred from measured geometric leaf features

\section{ACKNOWLEDGMENTS}

Thanks to the Computer Science and Information Technology graduate staff and students.

\section{REFERENCES}

[1] van der Heijden, G., de Visser, P., and Heuvelink, E. (2007). Functional-Structural Plant Modelling in Crop Production: Measurements for Functional-Structural Crop Models, chapter 2, pages $13\{25$. Springer.

[2] Chaerle, L. and Van Der Straeten, D. (2001). Seeing is believing: imaging techniques to monitor plant health. Biochimica et Biophysica Acta (BBA) - Gene Structure and Expression, 1519(3):153166.

[3] Romer, C., Wahabzada, M., Ballvora, A., Pinto, F., Rossini, M., Panigada, C., Behmann, J., Leon, J., Thurau, C., Bauckhage, C., Kersting, K., Rascher, U., and Plumer, L. (2012). Early drought stress detection in cereals: simplex volume maximisation for hyperspectral image analysis. Functional Plant Biology, 39:878 890.

[4] Russell, S. J. and Norvig, P. (2010). Artificial Intelligence: A Modern Approach. Pearson Education, 3rd edition.

[5] Uhrmann, F., Hugel, C., Paris, S., Scholz, O., Zollhofer, M., and Greiner, G. (2013). A model-based approach to extract leaf features from $3 \mathrm{~d}$ scans. In Proceedings of the 7th International Conference on Functional-Structural Plant Models, Saariselka, Finland.

[6] Mitchell, T. (1997). Machine Learning. McGraw-Hill International Editions.

[7] Han, J. and Kamber, M. (2005). Data mining: concepts and techniques. Kaufmann, San Francisco [u.a.].

[8] Alpaydin, E. (2004). Introduction to Machine Learning. Adaptive computation and machine learning. MIT Press.

[9] Camargo, A. and Smith, J. (2009a). Image pattern classification for the identification of disease-causing agents in plants. Computers and Electronics in Agriculture, 66:121\{125.

[10] Seatovic, D. (2008). 3d-object recognition, localization, and treatment of rumex obtusifoliol in its natural environment. In 1st International Conference on Machine Control \& Guidance.

[11] van der Heijden, G., de Visser, P., and Heuvelink, E. (2007). Functional-Structural Plant Modelling in Crop Production: Measurements for Functional-Structural Crop Models, chapter 2, pages $13\{25$. Springer.

[12] Paulus, S., Dupuis, J., Mahlein, A.-K., and Kuhlmann, H. (2013). Surface feature based classification of plant using machine learning techniques. The features were acquired using a measuring system developed at the Fraunhofer IIS. The aim was to evaluate which of the available features are required to model drought stress and whether they are salient to reach a reliable classification performance.

An overview of existing approaches and techniques with respect to vitality assessment of plants via remote sensors should be discussed.

organs from $3 \mathrm{~d}$ laser scanned point clouds for plant phenotyping. BMC Bioinformatics, 14:238.

[13] Balfer, J., Scholer, F., and Steinhage, V. (2013). Semantic skeletonization for structural plant analysis. In Proceedings of the 7th International Conference on Functional-Structural Plant Models, Saariselka, Finland.

[14] Dornbusch, T., Wernecke, P., and Diepenbrock, W. (2007). A method to extract morphological traits of plant organs from $3 \mathrm{~d}$ point clouds as a database for an architectural plant model. Ecological Modelling, 200:119\{129.

[15] Camargo, A. and Smith, J. (2009b). An image-processing based algorithm to automatically identify plant disease visual symptoms. Biosystems Engineering, 102, Issue 1:921.

[16] Mucherino, A., Papajorgji, P., and Pardalos, P. M. (2009). A survey of data mining techniques applied to agriculture. Operational Research, 9, Issue 2:121\{140.

[17] Rumpf, T., Mahlein, A.-K., Steiner, U., Oerke, E.-C., Dehne, H.-W., and Plumer, L. (2010). Early detection and classification of plant diseases with support vector machines based on hyperspectral reflectance. Computers and Electronics in Agriculture, 74:91\{99. 